\title{
Synchronization of Chaotic Fractional-Order WINDMI Systems via Linear State Error Feedback Control
}

\author{
Baogui Xin, ${ }^{1,2}$ Tong Chen, ${ }^{1}$ and Yanqin Liu ${ }^{1,3}$ \\ ${ }^{1}$ Nonlinear Dynamics and Chaos Group, School of Management, Tianjin University, \\ Tianjin 300072, China \\ ${ }^{2}$ Center for Applied Mathematics, School of Economics and Management, Shandong University of Science \\ and Technology, Qingdao 266510, China \\ ${ }^{3}$ Department of Mathematics, Dezhou University, Dezhou 253023, China
}

Correspondence should be addressed to Baogui Xin, xin@tju.edu.cn

Received 25 April 2010; Accepted 20 August 2010

Academic Editor: Yuri Vladimirovich Mikhlin

Copyright (C) 2010 Baogui Xin et al. This is an open access article distributed under the Creative Commons Attribution License, which permits unrestricted use, distribution, and reproduction in any medium, provided the original work is properly cited.

\begin{abstract}
We propose a fractional-order WINDMI system, as a generalization of an integer-order system developed by Sprott (2003). The considered synchronization scheme consists of identical master and slave fractional-order WINDMI systems coupled by linear state error variables. Based on the stability theory of nonlinear fractional-order systems, linear state error feedback control technique is applied to achieve chaos synchronization, and a linear control law is derived analytically to achieve synchronization of the chaotic fractional-order WINDMI system. Numerical simulations validate the main results of this work.
\end{abstract}

\section{Introduction}

The solar-wind-driven magnetosphere-ionosphere is a complex driven-damped dynamical system which exhibits a variety of dynamical states that include low-level steady plasma convection, episodic releases of geotail stored plasma energy into the ionosphere known broadly as substorms, and states of continuous strong unloading [1]. In 1998, Horton and Doxas [2] firstly proposed the WINDMI system, a six-dimensional nonlinear dynamics model, which was derived for the basic energy components of the night-side magnetotail coupled to the ionosphere by the region-1 currents. Smith et al. [3] explored the dynamical range of the WINDMI model. Horton et al. [1] introduced reductions to derive a new minimal three-dimensional WINDMI model. Sprott [4] further simplified the integer-order WINDMI 


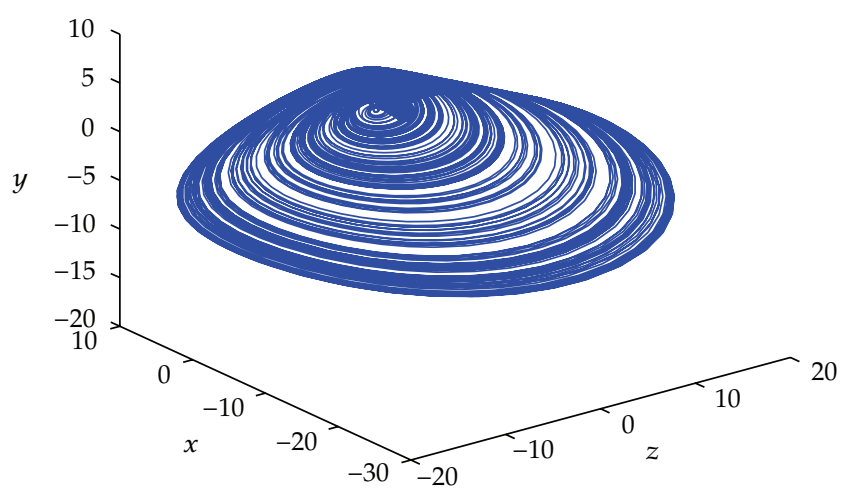

Figure 1: Phase portrait of system (1.1) versus $a=0.7$ and $b=2.5$.

model as follows:

$$
\begin{aligned}
& \frac{d x}{d t}=y, \\
& \frac{d y}{d t}=z, \\
& \frac{d z}{d t}=-a z-y+b-e^{x}
\end{aligned}
$$

where $x, y$, and $z$ are variables and $a, b$ are positive constants. System (1.1) has a chaotic attractor when the usual parameters are $a=0.7$ and $b=2.5$, as shown in Figure 1 (see [4]).

Fractional calculus, a generalization of differentiation and integration to an arbitrary order, is an old mathematical topic with over 300-year-old history [5]. Fractional-order differential equations can be used to describe many systems in interdisciplinary fields, but it was not used in science and engineering for many years because there exist many difficulties, such as the absence of impactful solution methods and numerical simulation schemes for fractional differential equations. However, during the past several years, with the development of computer simulation technology $[6,7]$, fractional calculus has been realizing the utility and applicability to various branches of science and engineering, such as fractional viscoelastic fluids [8], fractional diffusion processes [9, 10], fractional-order viscoelastic material models [11], fractional-order HIV Model [12, 13], and fractional-order controllers [14-16]. Undoubtedly, fractional calculus will be applied into more and more areas of classical and modern analysis.

Chaos synchronization plays a very important role in the theory and applications. Synchronization of fractional-order chaotic systems was first presented by Deng and Li [17]. As an active research area, chaos synchronization with fractional calculus has received increasing attention in recent years due to its potentials in both theory and applications [18-25]. On the other hand, as compared with sliding mode control, standard PID feedback control, and so on, the advantage of linear state error feedback control is that it is linear and easier to implement for chaos synchronization.

The remainder of this paper is organized as follows. In Section 2, the WINDMI system is generalized from integer to noninteger order. Then, chaos synchronization via linear 
feedback control is studied in Section 3. Numerical simulations are presented in Section 4, and finally conclusions in Section 5 close the paper.

\section{System Description}

There are several definitions of fractional derivatives. In this paper, we will adopt the Riemann-Liouville fractional derivative definition as follows.

Definition 2.1 (see [26]). The $q$ th-order fractional derivative of function $f(t)$ with respect to $t$ and the terminal value 0 is given by

$$
\frac{d^{q} f(t)}{d t^{q}}=\frac{1}{\Gamma(m-q)} \frac{d^{m}}{d t^{m}} \int_{0}^{t} \frac{f(\tau)}{(t-\tau)^{q-m+1}} d \tau
$$

where $\Gamma(\cdot)$ is a Gamma function, $m$ is an integer and satisfies $m-1<q<m \in Z^{+}$.

The integer derivative of a function has relationship with only its nearby points while the fractional derivative takes into account nonlocal characteristics like "infinite memory" $[26,27]$. As a result, a model described with fractional derivative possesses memory, which may help to have a better understanding to the importance of assembling large substorm databases with a large range of event sizes. Here, we introduce fractional calculus into system (1.1). The new system is described with fractional derivative as follows:

$$
\begin{aligned}
& \frac{d^{q_{1}} x}{d t^{q_{1}}}=y, \\
& \frac{d^{q_{2}} y}{d t^{q_{2}}}=z, \\
& \frac{d^{q_{3}} z}{d t^{q_{3}}}=-a z-y+b-e^{x},
\end{aligned}
$$

in which $x, y$, and $z$ are variables and $a, b$ are positive constants; $q=\left(q_{1}, q_{2}, q_{3}\right)$ is subject to $0<q_{1}, q_{2}, q_{3}<1$. If $q=(1,1,1)$, system (2.2) degenerates into system (1.1).

In order to observe the synchronization behavior in two identical fractional-order WINDMI systems, we set a drive-response configuration with a drive system given by the fractional-order WINDMI systems (with three state variables denoted by the subscript $m$ ) and with a response system (with three variables denoted by the subscript $s$ ) as follows.

The drive system is described by

$$
\begin{aligned}
& \frac{d^{q_{1}} x_{m}}{d t^{q_{1}}}=y_{m}, \\
& \frac{d^{q_{2}} y_{m}}{d t^{q_{2}}}=z_{m}, \\
& \frac{d^{q_{3}} z_{m}}{d t^{q_{3}}}=-a z_{m}-y_{m}+b-e^{x_{m}},
\end{aligned}
$$


and the response system is given by

$$
\begin{aligned}
& \frac{d^{q_{1}} x_{s}}{d t^{q_{1}}}=y_{s}+u_{1}, \\
& \frac{d^{q_{2}} y_{s}}{d t^{q_{2}}}=z_{s}+u_{2}, \\
& \frac{d^{q_{3}} z_{s}}{d t^{q_{3}}}=-a z_{s}-y_{s}+b-e^{x_{s},}
\end{aligned}
$$

where $u_{1}$ and $u_{2}$ are the linear state error feedback controllers. Then, the rest of our task is to design suitable linear controllers that can synchronize systems (2.3) and (2.4).

\section{Synchronization Scheme}

Matignon [28] defined the internal and external stability properties of linear fractional-order differential systems of finite dimension and derived the necessary and sufficient conditions.

Theorem 3.1 (see [28]). Consider that the following $n$-dimensional linear fractional-order autonomous system:

$$
\frac{d^{q} x}{d t^{q}}=A x, \quad x(0)=x_{0}
$$

with $0<q<1, x \in R^{n}$, and $A \in R^{n \times n}$, is asymptotically stable if and only if $|\arg (\lambda)|>q \pi / 2$ is satisfied for all eigenvalues $\lambda$ of matrix A. Also system (3.1) is stable if and only if $|\arg (\lambda)| \geq q \pi / 2$ is satisfied for all eigenvalues $\lambda$ of matrix $A$.

Theorem 3.2 (see [29]). Consider the following autonomous n-dimensional nonlinear fractional differential equation:

$$
\frac{d^{q} X(t)}{d t^{q}}=F(X(t)), \quad X(0)=X_{0}=\left(x_{10}, x_{20}, \ldots, x_{n 0}\right)^{T}, \quad m-1<q<m \in Z^{+} .
$$

Letting $\widehat{X}=\left(\widehat{x}_{1}, \widehat{x}_{2}, \ldots, \widehat{x}_{n}\right)^{T}$ be an equilibrium of system (3.2), that is, $d^{q} \hat{X} / d t^{q}=F(X)=0$, and letting $A=\left.(\partial F / \partial X)\right|_{X=\widehat{X}}$ be the Jacobian matrix at the point $\widehat{X}$, then the point $\hat{X}$ is asymptotically stable when $|\arg (e i g(A))|>q_{m} \pi / 2$, where $q_{m}=\max _{1 \leq i \leq n}\left\{q_{i}\right\}$.

From Theorem 3.2, the following corollary holds.

Corollary 3.3. Letting $\widehat{X}=\left(\widehat{x}_{1}, \widehat{x}_{2}, \ldots, \widehat{x}_{n}\right)^{T}$ be an equilibrium of system (3.2), that is, $d^{q} \hat{X} / d t^{q}=$ $F(\widehat{X})=0$, and letting $A=\left.(\partial F / \partial X)\right|_{X=\widehat{X}}$ be the Jacobian matrix at the point $\widehat{X}$, then the point $\widehat{X}$ is locally asymptotically stable if $A$ is an upper or lower triangular matrix and all eigenvalues of $A$ are negative real numbers. 
Theorem 3.4. The drive system (2.3) and the response system (2.4) will approach global synchronization for any initial condition with the following control law:

$$
u_{1}=c_{1}\left(x_{s}-x_{m}\right)-\left(y_{s}-y_{m}\right), \quad u_{2}=c_{2}\left(y_{s}-y_{m}\right)-\left(z_{s}-z_{m}\right),
$$

where $c_{1}<0$ and $c_{2}<0$.

Proof. Set the synchronization error variables as follows:

$$
\begin{aligned}
& e_{1}=x_{s}-x_{m}, \\
& e_{2}=y_{s}-y_{m}, \\
& e_{3}=z_{s}-z_{m} .
\end{aligned}
$$

By subtracting (2.3) from (2.4) and using (3.4), the synchronization error system can be obtained as follows:

$$
\begin{aligned}
& \frac{d^{q_{1}} e_{1}}{d t^{q_{1}}}=e_{2}+u_{1}, \\
& \frac{d^{q_{2}} e_{2}}{d t^{q_{2}}}=e_{3}+u_{2}, \\
& \frac{d^{q_{3}} e_{3}}{d t^{q_{3}}}=-a e_{3}-e_{2}+e^{x_{m}}\left(1-e^{e_{1}}\right),
\end{aligned}
$$

where $a>0, x_{m}$ is the state variable of system (2.3), $u_{1}$ and $u_{2}$ are linear state error feedback controllers.

When $u_{1}=c_{1}\left(x_{s}-x_{m}\right)-\left(y_{s}-y_{m}\right)$ and $u_{2}=c_{2}\left(y_{s}-y_{m}\right)-\left(z_{s}-z_{m}\right)$, system (3.5) can be rewritten as

$$
\begin{aligned}
& \frac{d^{q_{1}} e_{1}}{d t^{q_{1}}}=c_{1} e_{1}, \\
& \frac{d^{q_{2}} e_{2}}{d t^{q_{2}}}=c_{2} e_{2}, \\
& \frac{d^{q_{3}} e_{3}}{d t^{q_{3}}}=-a e_{3}-e_{2}+e^{x_{m}}\left(1-e^{e_{1}}\right) .
\end{aligned}
$$

System (3.6) has only one equilibrium point at $E_{0}=(0,0,0)$. Its Jacobian matrix evaluated at equilibrium point $E_{0}$ is given by

$$
J\left(E_{0}\right)=\left(\begin{array}{ccc}
c_{1} & 0 & 0 \\
0 & c_{2} & 0 \\
-e^{x_{m}+e_{1}} & -1 & -a
\end{array}\right)=\left(\begin{array}{ccc}
c_{1} & 0 & 0 \\
0 & c_{2} & 0 \\
-e^{x_{m}} & -1 & -a
\end{array}\right)
$$

Obviously, (3.7) is a lower triangular matrix, and $x_{m}$ is a state variable of drive system (2.3). Thus, from Corollary 3.3, one can get that $x_{m}$ has no effect on the stability of system 


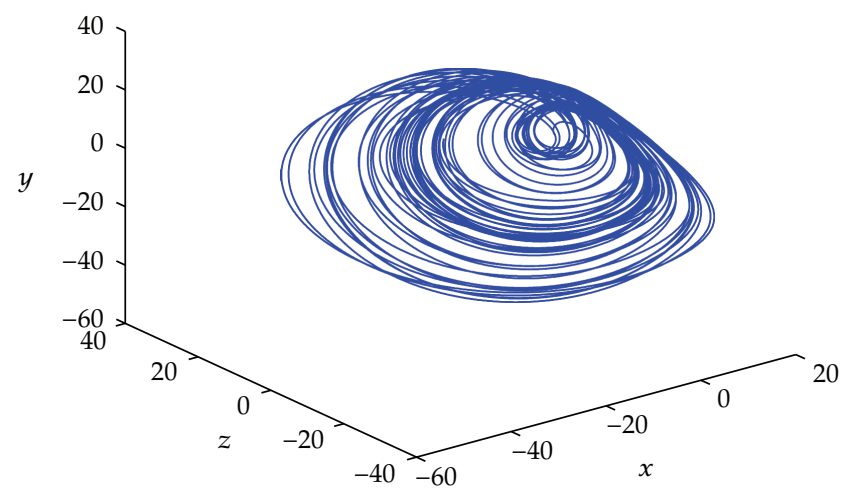

(a) Drive system (2.3)

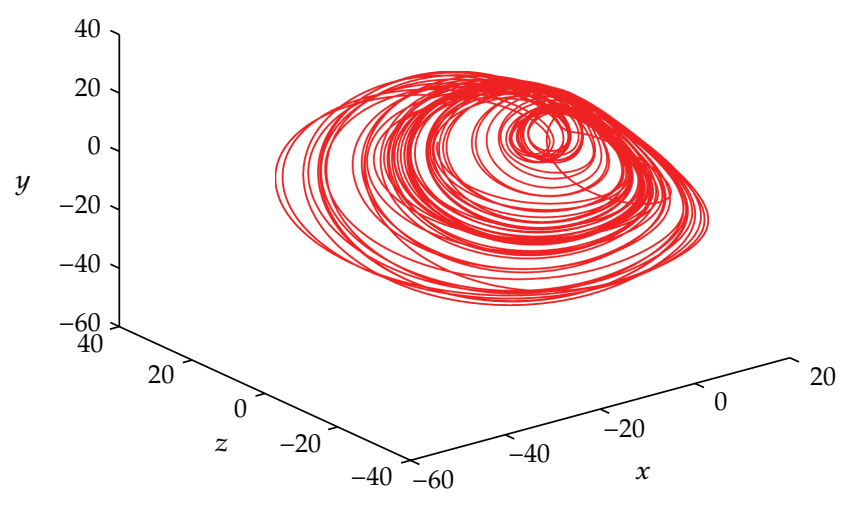

(b) Response system (2.4)

Figure 2: Chaotic attractors of drive system (2.3) and response system (2.4) versus $q_{1}=0.77, q_{2}=$ $0.98, q_{3}=0.8, a=0.8, b=6.4, c_{1}=-1$, and $c_{2}=-1$.

(3.6), and if $c_{1}<0$ and $c_{2}<0$, then system (3.6) is asymptotically stable; that is, the drive system (2.3) and the response system (2.4) are synchronized finally.

The theorem is proved.

Evidently, the advantage of the linear feedback controllers proposed in Theorem 3.4 is that they are robust, linear, and have lower dimensions than that of the states; morever, they are easier to be designed and implemented for chaos synchronization than standard PID feedback controllers, sliding mode controllers, nonlinear feedback controllers, and so on.

\section{Numerical Simulations}

\subsection{Discretization Scheme}

Based on the Adams-Bashforth-Moulton predictor-corrector scheme [6, 30], we can build the numerical calculation formula for the proposed synchronization scheme as follows. 
Letting $(x, y, z)$ and $(\widehat{x}, \hat{y}, \widehat{z})$ represent $\left(x_{m}, y_{m}, z_{m}\right)$ and $\left(x_{s}, y_{s}, z_{s}\right)$, respectively. Setting $h=T / N, t_{n}=n h, n=0,1, \ldots, N \in Z^{+}$, the drive system (2.3) and the response system (2.4) can be discretized as

$$
\begin{aligned}
& x_{n+1}=x_{0}+\frac{h^{q_{1}}}{\Gamma\left(q_{1}+2\right)} y_{n+1}^{p}+\frac{h^{q_{1}}}{\Gamma\left(q_{1}+2\right)} \sum_{j=0}^{n} \alpha_{1, j, n+1} y_{j}, \\
& y_{n+1}=y_{0}+\frac{h^{q_{2}}}{\Gamma\left(q_{2}+2\right)} z_{n+1}^{p}+\frac{h^{q_{2}}}{\Gamma\left(q_{2}+2\right)} \sum_{j=0}^{n} \alpha_{2, j, n+1} z_{j}, \\
& z_{n+1}=z_{0}+\frac{h^{q_{3}}}{\Gamma\left(q_{3}+2\right)}\left(-e^{x_{n+1}^{p}}-y_{n+1}^{p}-a z_{n+1}^{p}+b\right)+\frac{h^{q_{3}}}{\Gamma\left(q_{3}+2\right)} \sum_{j=0}^{n} \alpha_{3, j, n+1}\left(-e^{x_{j}}-y_{j}-a z_{j}+b\right), \\
& \widehat{x}_{n+1}=\widehat{x}_{0}+\frac{h^{q_{1}}}{\Gamma\left(q_{1}+2\right)}\left(y_{n+1}^{p}+c_{1}\left(\widehat{x}_{n+1}^{p}-x_{n+1}^{p}\right)\right)+\frac{h^{q_{1}}}{\Gamma\left(q_{1}+2\right)} \sum_{j=0}^{n} \alpha_{1, j, n+1}\left(y_{j}+c_{1}\left(\widehat{x}_{j}-x_{j}\right)\right), \\
& \widehat{y}_{n+1}=\widehat{y}_{0}+\frac{h^{q_{2}}}{\Gamma\left(q_{2}+2\right)}\left(z_{n+1}^{p}+c_{2}\left(\widehat{y}_{n+1}^{p}-y_{n+1}^{p}\right)\right)+\frac{h^{q_{2}}}{\Gamma\left(q_{2}+2\right)} \sum_{j=0}^{n} \alpha_{2, j, n+1}\left(z_{j}+c_{2}\left(\widehat{y}_{j}-y_{j}\right)\right), \\
& \widehat{z}_{n+1}=\widehat{z}_{0}+\frac{h^{q_{3}}}{\Gamma\left(q_{3}+2\right)}\left(-e^{\hat{x}_{n+1}^{p}}-\widehat{y}_{n+1}^{p}-a \widehat{z}_{n+1}^{p}+b\right)+\frac{h^{q_{3}}}{\Gamma\left(q_{3}+2\right)} \sum_{j=0}^{n} \alpha_{3, j, n+1}\left(-e^{\widehat{x}_{j}}-\widehat{y}_{j}-a \widehat{z}_{j}+b\right),
\end{aligned}
$$

where

$$
\begin{aligned}
& x_{n+1}^{p}=x_{0}+\frac{1}{\Gamma\left(q_{1}\right)} \sum_{j=0}^{n} \beta_{1, j, n+1} y_{j}, \\
& y_{n+1}^{p}=y_{0}+\frac{1}{\Gamma\left(q_{2}\right)} \sum_{j=0}^{n} \beta_{2, j, n+1} z_{j}, \\
& z_{n+1}^{p}=z_{0}+\frac{1}{\Gamma\left(q_{3}\right)} \sum_{j=0}^{n} \beta_{3, j, n+1}\left(-e^{x_{j}}-y_{j}-a z_{j}+b\right), \\
& \hat{x}_{n+1}^{p}=\widehat{x}_{0}+\frac{1}{\Gamma\left(q_{1}\right)} \sum_{j=0}^{n} \beta_{1, j, n+1}\left(y_{j}+c_{1}\left(\widehat{x}_{j}-x_{j}\right)\right), \\
& \hat{y}_{n+1}^{p}=\widehat{y}_{0}+\frac{1}{\Gamma\left(q_{2}\right)} \sum_{j=0}^{n} \beta_{2, j, n+1}\left(z_{j}+c_{2}\left(\widehat{y}_{j}-y_{j}\right)\right), \\
& \hat{z}_{n+1}^{p}=\widehat{z}_{0}+\frac{1}{\Gamma\left(q_{3}\right)} \sum_{j=0}^{n} \beta_{3, j, n+1}\left(-e^{\widehat{x}_{j}}-\widehat{y}_{j}-a \widehat{z}_{j}+b\right),
\end{aligned}
$$




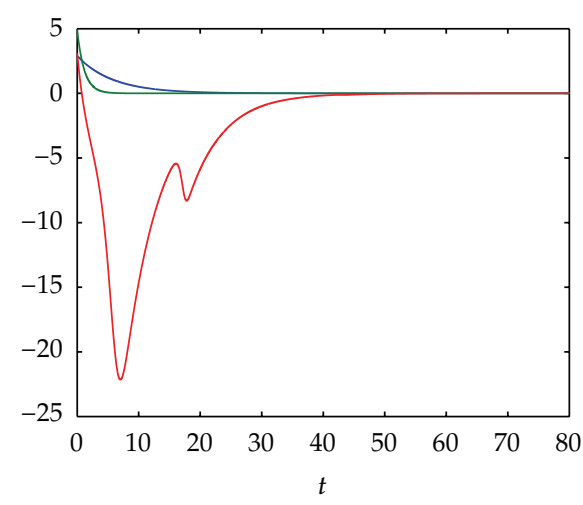

$$
\begin{array}{r}
e_{1} \\
-e_{2} \\
-e_{3}
\end{array}
$$

(a) Synchronization errors between drive system (2.3) and response system (2.4)
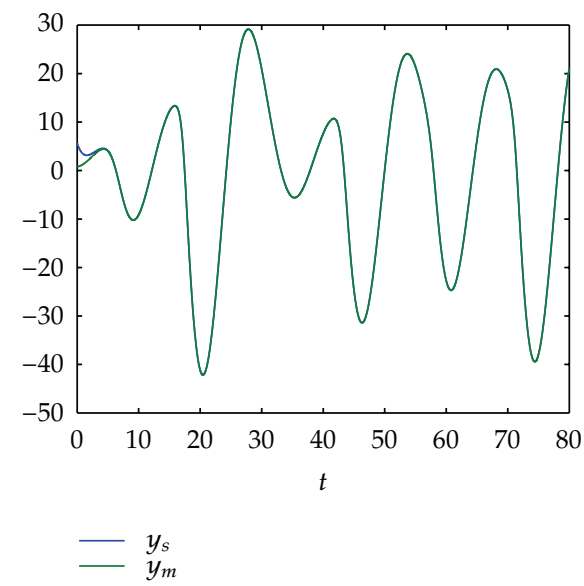

(c) Time evolutions of $y_{s}$ and $y_{m}$
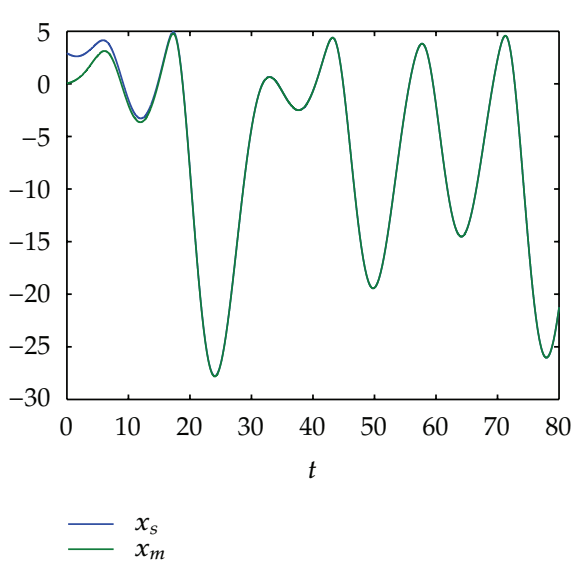

(b) Time evolutions of $x_{s}$ and $x_{m}$

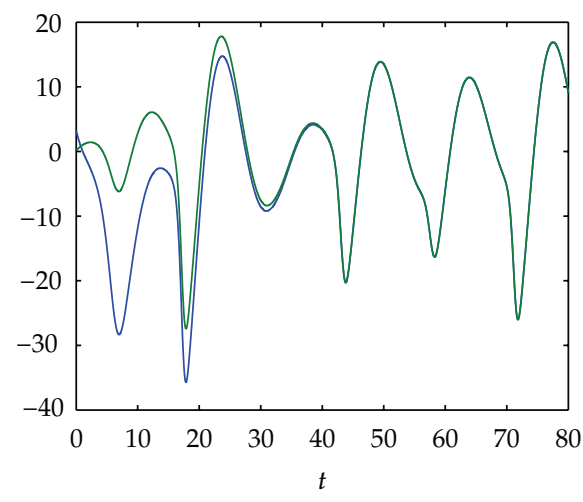

$-z_{s}$

(d) Time evolutions of $z_{s}$ and $z_{m}$

Figure 3: Synchronization errors between drive system (2.3) and response system (2.4) versus $q_{1}=$ $0.77, q_{2}=0.98, q_{3}=0.8, a=0.8, b=6.4, c_{1}=-1$, and $c_{2}=-1$.

$$
\begin{aligned}
& \alpha_{i, j, n+1}= \begin{cases}n^{q_{i}+1}-\left(n-q_{i}\right)(n+1)^{q_{i}}, & j=0, \\
(n-j+2)^{q_{i}+1}+(n-j)^{q_{i}+1}-2(n-j+1)^{q_{i+1},}, & 1 \leq j \leq n, i=1,2,3, \\
1, & j=n+1,\end{cases} \\
& \beta_{i, j, n+1}=\frac{h^{q_{i}}}{q_{i}}\left((n-j+1)^{q_{i}}-(n-j)^{q_{i}}\right), \quad 0 \leq j \leq n, i=1,2,3 .
\end{aligned}
$$




\subsection{Numerical Results}

Based on the above mentioned discretization scheme, the drive system (2.3) and the response system (2.4) are integrated numerically with the fractional orders $q_{1}=0.77, q_{2}=0.98, q_{3}=$ 0.8 and using the initial values $x_{m}(0)=0, y_{m}(0)=0.8, z_{m}(0)=0, x_{s}(0)=4, y_{s}(0)=4.8$, and $z_{s}(0)=4$. Let $a=0.8, b=6.4, c_{1}=-1, c_{2}=-1$; the chaotic attractor of the drive system (2.3) is shown in Figure 2(a), and the chaotic attractor of the response system (2.4) is shown in Figure 2(b). From Figures 3(a)-3(d), it is clear that the synchronization is achieved for all these values.

\section{Conclusion}

In this paper, we introduce fractional-order calculus into the WINDMI system. Chaos synchronization of identical master and slave fractional-order WINDMI systems is studied by utilizing linear state error feedback control technique. Based on the stability theory of nonlinear fractional-order systems, linear feedback control law for chaos synchronization has been investigated. Numerical simulations are given to verify the effectiveness of the proposed synchronization scheme.

\section{Acknowledgments}

The authors are very grateful for the editor in charge Yuri Vladimirovich Mikhlin for the efficient editorial work. The insightful suggestions from the referees improved the quality and the readability substantially. This paper is supported partly by the China Postdoctoral Science Foundation (Grant no. 20100470783), Specialized Research Fund for Doctoral Program of Higher Education from Ministry of Education of China (Grant no. 20090032110031), and National Natural Science Foundation of China (Grant no. 60904063).

\section{References}

[1] W. Horton, R. S. Weigel, and J. C. Sprott, "Chaos and the limits of predictability for the solar-winddriven magnetosphere-ionosphere system," Physics of Plasmas, vol. 8, no. 6, pp. 2946-2952, 2001.

[2] W. Horton and I. Doxas, "A low-dimensional dynamical model for the solar wind driven geotailionosphere system," Journal of Geophysical Research A, vol. 103, no. A3, pp. 4561-4572, 1998.

[3] J. P. Smith, J.-L. Thiffeault, and W. Horton, "Dynamical range of the WINDMI model: an exploration of possible magnetospheric plasma states," Journal of Geophysical Research A, vol. 105, no. A6, pp. 12983-12996, 2000.

[4] J. C. Sprott, Chaos and Time-Series Analysis, Oxford University Press, New York, NY, USA, 2003.

[5] K. B. Oldham and J. Spanier, The Fractional Calculus. Theory and Applications of Differentiation and Integration to Arbitrary Order, Academic Press, New York, NY, USA, 1974.

[6] K. Diethelm, N. J. Ford, and A. D. Freed, "A predictor-corrector approach for the numerical solution of fractional differential equations," Nonlinear Dynamics, vol. 29, no. 1-4, pp. 3-22, 2002.

[7] W. Deng, "Numerical algorithm for the time fractional Fokker-Planck equation," Journal of Computational Physics, vol. 227, no. 2, pp. 1510-1522, 2007.

[8] S. Wang and M. Xu, "Axial Couette flow of two kinds of fractional viscoelastic fluids in an annulus," Nonlinear Analysis. Real World Applications, vol. 10, no. 2, pp. 1087-1096, 2009.

[9] X. Jiang, M. Xu, and H. Qi, "The fractional diffusion model with an absorption term and modified Fick's law for non-local transport processes," Nonlinear Analysis. Real World Applications, vol. 11, no. 1, pp. 262-269, 2010.

[10] Y.-Q. Liu and J.-H. Ma, “Exact solutions of a generalized multi-fractional nonlinear diffusion equation in radical symmetry," Communications in Theoretical Physics, vol. 52, no. 5, pp. 857-861, 2009. 
[11] K. D. Papoulia, V. P. Panoskaltsis, N. V. Kurup, and I. Korovajchuk, "Rheological representation of fractional order viscoelastic material models," Rheologica Acta, vol. 49, no. 4, pp. 381-400, 2010.

[12] H. Ye and Y. Ding, "Nonlinear dynamics and chaos in a fractional-order HIV model," Mathematical Problems in Engineering, vol. 2009, Article ID 378614, 12 pages, 2009.

[13] Y. Ding and H. Ye, "A fractional-order differential equation model of HIV infection of CD4 ${ }^{+}$T-cells," Mathematical and Computer Modelling, vol. 50, no. 3-4, pp. 386-392, 2009.

[14] R. E. Gutiérrez, J. M. Rosário, and J. Tenreiro MacHado, "Fractional order calculus: basic concepts and engineering applications," Mathematical Problems in Engineering, vol. 2010, Article ID 375858, 19 pages, 2010.

[15] I. Podlubny, "Fractional-order systems and $P I^{\lambda} D^{\mu}$-controllers," IEEE Transactions on Automatic Control, vol. 44, no. 1, pp. 208-214, 1999.

[16] J. A. Tenreiro Machado, M. F. Silva, R. S. Barbosa et al., "Some applications of fractional calculus in engineering," Mathematical Problems in Engineering, vol. 2010, Article ID 639801, 34 pages, 2010.

[17] W. H. Deng and C. P. Li, "Chaos synchronization of the fractional Lü system," Physica A, vol. 353, no. 1-4, pp. 61-72, 2005.

[18] C. P. Li, W. H. Deng, and D. Xu, "Chaos synchronization of the Chua system with a fractional order," Physica A, vol. 360, no. 2, pp. 171-185, 2006.

[19] X.-Y. Wang and J.-M. Song, "Synchronization of the fractional order hyperchaos Lorenz systems with activation feedback control," Communications in Nonlinear Science and Numerical Simulation, vol. 14, no. 8, pp. 3351-3357, 2009.

[20] X. Y. Wang and Y. He, "Projective synchronization of fractional order chaotic system based on linear separation," Physics Letters A, vol. 372, no. 4, pp. 435-441, 2008.

[21] R.-X. Zhang, S.-P. Yang, and Y.-L. Liu, "Synchronization of fractional-order unified chaotic system via linear control," Acta Physica Sinica, vol. 59, no. 3, pp. 1549-1553, 2010.

[22] H. Zhu, S. Zhou, and Z. He, "Chaos synchronization of the fractional-order Chen's system," Chaos, Solitons and Fractals, vol. 41, no. 5, pp. 2733-2740, 2009.

[23] Z. M. Odibat, N. Corson, M. A. Aziz-Alaoui, and C. Bertelle, "Synchronization of chaotic fractionalorder systems via linear control," International Journal of Bifurcation and Chaos, vol. 20, no. 1, pp. 81-97, 2010.

[24] X. Wu, H. Lu, and S. Shen, "Synchronization of a new fractional-order hyperchaotic system," Physics Letters A, vol. 373, no. 27-28, pp. 2329-2337, 2009.

[25] M. Shahiri, R. Ghaderi, A. Ranjbar N., S. H. Hosseinnia, and S. Momani, "Chaotic fractional-order Coullet system: synchronization and control approach," Communications in Nonlinear Science and Numerical Simulation, vol. 15, no. 3, pp. 665-674, 2010.

[26] I. Podlubny, Fractional Differential Equations. An Introduction to Fractional Derivatives, Fractional Differential Equations, to Methods of Their Solution and Some of Their Applications, vol. 198 of Mathematics in Science and Engineering, Academic Press, San Diego, Calif, USA, 1999.

[27] D. Cafagna, "Past and present-fractional calculus: a mathematical tool from the past for present engineers," IEEE Industrial Electronics Magazine, vol. 1, no. 2, pp. 35-40, 2007.

[28] D. Matignon, "Stability results for fractional differential equations with applications to control processing," in Proceedings of the IMACS-IEEE Multiconference on Computational Engineering in Systems Applications (CESA '96), pp. 963-968, Lille, France, 1996.

[29] Y. Yu, H.-X. Li, S. Wang, and J. Yu, "Dynamic analysis of a fractional-order Lorenz chaotic system," Chaos, Solitons and Fractals, vol. 42, no. 2, pp. 1181-1189, 2009.

[30] K. Diethelm, N. J. Ford, and A. D. Freed, "Detailed error analysis for a fractional Adams method," Numerical Algorithms, vol. 36, no. 1, pp. 31-52, 2004. 


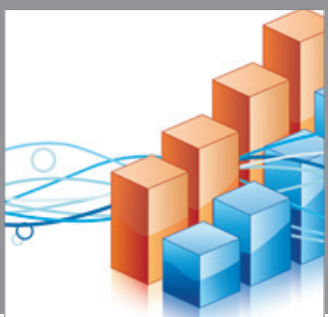

Advances in

Operations Research

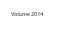

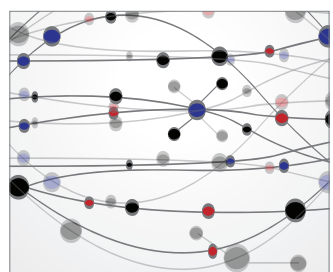

\section{The Scientific} World Journal
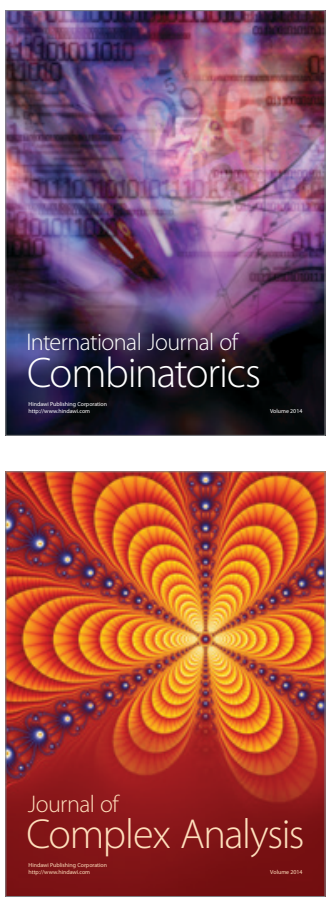

International Journal of

Mathematics and

Mathematical

Sciences
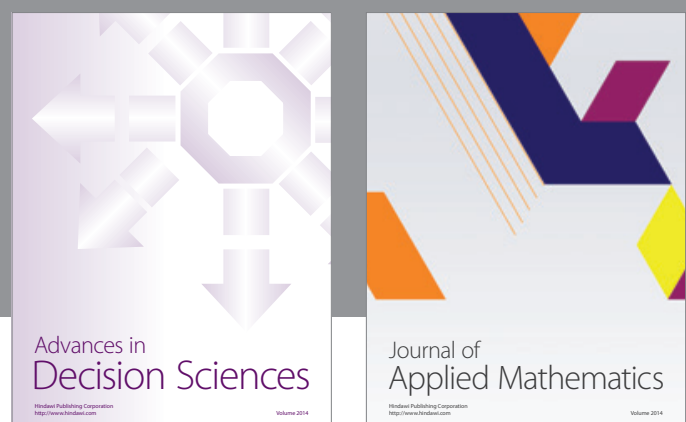

Journal of

Applied Mathematics
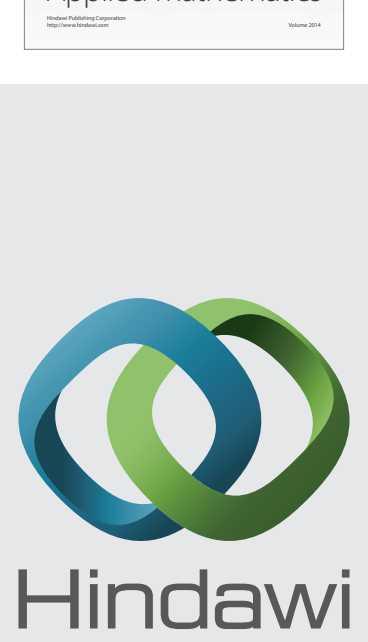

Submit your manuscripts at http://www.hindawi.com
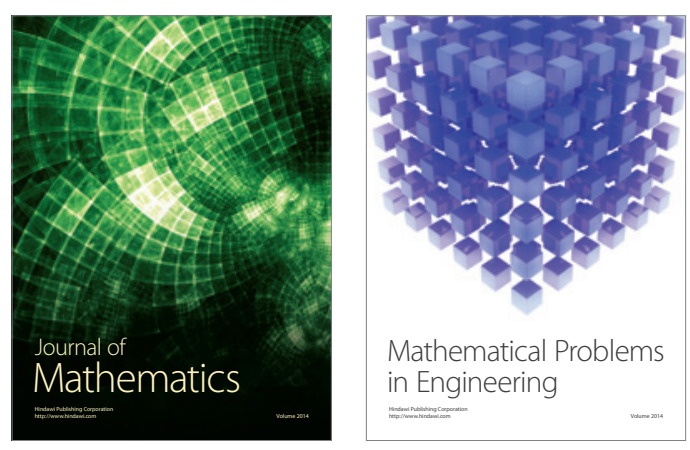

Mathematical Problems in Engineering
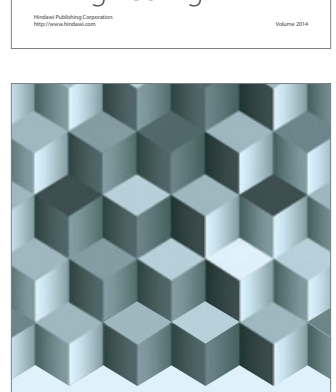

Journal of

Function Spaces
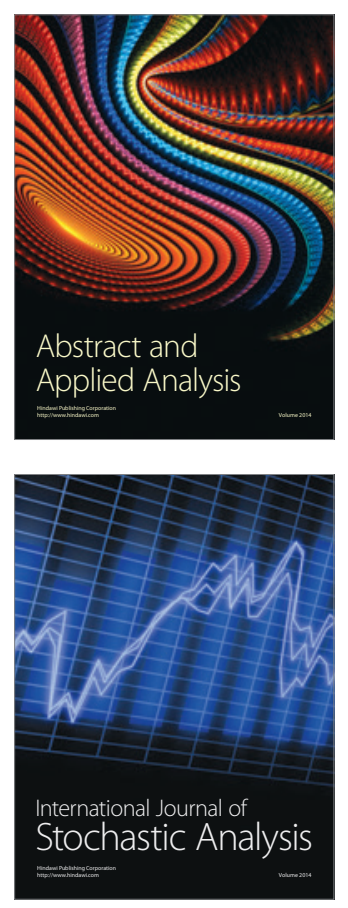

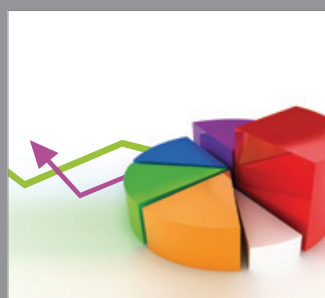

ournal of

Probability and Statistics

Promensencen
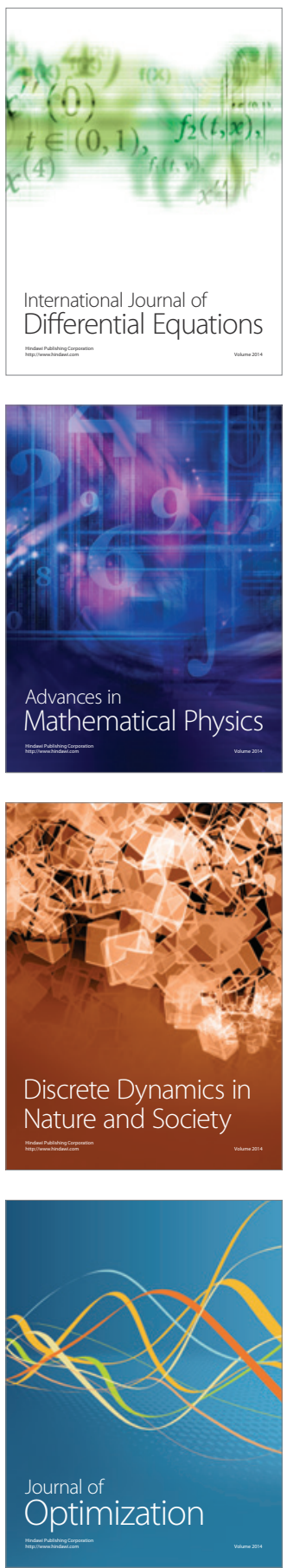